INPLASY

PROTOCOL

To cite: Liu et al. Efficacy and safety of Tian Meng oral liquid for the treatment of insomnia A protocol for systematic review and meta-analysis. Inplasy protocol 202170035. doi: 10.37766/inplasy2021.7.0035

Received: 12 July 2021

Published: 12 July 2021

Corresponding author:

Yuanxiang Liu

lyxlwtg@126.com

Author Affiliation:

Shandong University of traditional Chinese Medicine

Support: None.

Review Stage at time of this submission: The review has not yet started.

Conflicts of interest:

None declared.

\section{Efficacy and safety of Tian Meng oral liquid for the treatment of insomnia A protocol for systematic review and meta-analysis}

Liu, X1; Zhang, L2; Li, R3; Li, B4; Ge, X5; Liu, Y6; Yang, J7.

Review question / Objective: This study aims to evaluate the efficacy and safety of Tian Meng oral liquid in the treatment of insomnia and to provide a more reasonable option for clinical medication.

Condition being studied: The incidence of insomnia is very high and seriously affects the lives of its patients. Long-term insomnia can induce other diseases and even cause sudden death. Tian Meng oral liquid is a type of Chinese patent medicine that has been used in the clinical treatment of insomnia and has certain clinical effects. However, its wide application is limited by the lack of evidence-based medical evaluation.

INPLASY registration number: This protocol was registered with the International Platform of Registered Systematic Review and Meta-Analysis Protocols (INPLASY) on 12 July 2021 and was last updated on 12 July 2021 (registration number INPLASY202170035).

\section{INTRODUCTION}

Review question / Objective: This study aims to evaluate the efficacy and safety of Tian Meng oral liquid in the treatment of insomnia and to provide a more reasonable option for clinical medication.
Condition being studied: The incidence of insomnia is very high and seriously affects the lives of its patients. Long-term insomnia can induce other diseases and even cause sudden death. Tian Meng oral liquid is a type of Chinese patent medicine that has been used in the clinical treatment of insomnia and has certain clinical effects. 
However, its wide application is limited by the lack of evidence-based medical evaluation.

\section{METHODS}

Participant or population: Regardless of age or sex, patients met the diagnostic criteria for insomnia in the 2017 "Guidelines for the Diagnosis and Treatment of Insomnia in Adults in China".

Intervention: The treatment group was given Tian Meng oral liquid on the basis of conventional treatment.

Comparator: The control group was given only conventional treatment. Conventional treatment refers to sedation and oral medications such as zopiclone, dexzopiclone, alprazolam, estazolam, and clonazepam.

Study designs to be included: The included studies were clinical randomized controlled trials in this systematic review.

Eligibility criteria: The PICOS principles were given full consideration to establish the inclusion and exclusion criteria of this systematic review. Regardless of age or sex, patients met the diagnostic criteria for insomnia in the 2017 "Guidelines for the Diagnosis and Treatment of Insomnia in Adults in China".

Information sources: Studies were obtained from the China National Knowledge Infrastructure (CNKI), Wan Fang Data, Chinese Scientific Journals Database (VIP), PubMed, Embase, and Cochrane Library, regardless of publication date or language.

Main outcome(s): The main results included falling asleep time, sleep duration, sleep quality, physical and mental fatigue after waking up, daytime function, Pittsburgh Sleep Quality Index (PSQI) score, fatigue scale-14 (FS-14) score, and adverse events incidence rate.

Quality assessment / Risk of bias analysis: Two researchers assessed the quality of the included RCTs independently by utilizing the Cochrane risk of bias assessment tool.The two reviewers resolved any disagreements through discussion, and a third reviewer was involved if a consensus could not be reached.

Strategy of data synthesis: The metaanalysis was performed with Review Manager 5.3 and STATA $\mathbf{1 4 . 2}$ software. The outcomes were mainly represented by the mean difference (MD) or odds ratio (OR) with $95 \%$ confidence intervals $(\mathrm{Cl})$, and a $P$ value $<.05$ was considered significant. The Cochrane Q-test and 12 statistics were used to assess heterogeneity. When P50\% indicated statistical heterogeneity ,a random effects model was used to calculate the outcomes; otherwise, the fixed effect model was considered.

Subgroup analysis: If there was high heterogeneity in the studies, we performed subgroup analyses to explore the differences in age, sex, interventions, and course of disease/treatment.

Sensitivity analysis: To ensure robustness of the combined results, sensitivity analyses were performed to assess the impact of studies with a high risk of bias. We compared the results to determine whether low-quality studies should be excluded.

Country(ies) involved: China.

Keywords: traditional Chinese medicine; insomnia; protocol; systematic review; meta-analysis; Tian Meng oral liquid.

Contributions of each author:

Author 1 - Xinyu Liu.

Author 2 - Lu Zhang.

Author 3 - Runmin Li.

Author 4 - Bingchen Li.

Author 5 - Xin Ge.

Author 6 - Yuanxiang Liu.

Author 7 - Jiguo Yang. 